\title{
Vital role of entropy in photochirogenesis*
}

\author{
Yoshihisa Inoue ${ }^{1,2, \dagger}$, Norimitsu Sugahara ${ }^{2}$, and Takehiko Wada ${ }^{2}$ \\ ${ }^{1}$ Inoue Photochirogenesis Project, ERATO, JST, 4-6-3 Kamishinden, Toyonaka \\ 565-0085, Japan
}

\begin{abstract}
The effects of temperature, pressure, solvent, and substrate concentration upon several enantio- and diastereodifferentiating photochemical reactions have been investigated and discussed in detail. These entropy-related external factors, which manipulate the weak electronic and steric interactions in the excited state, dynamically control the stereochemical outcome of asymmetric photochemical reactions, and even switch the product chirality. A global view on the "entropy chemistry" and its potential applications are also discussed.
\end{abstract}

\section{INTRODUCTION}

Photochirogenesis, or photochemical generation of molecular chirality, is a relatively new area of chemistry that is rapidly growing, not only as a simple alternative to thermochemical or enzymatic asymmetric synthesis but also as a unique methodology for effecting asymmetric induction through the electronically excited state [1-5]. Photochemical asymmetric induction in the solution phase may be classified into three categories on the basis of the chiral source employed; the chiral interaction can take place between: 1) chiral light and molecule, 2) chiral molecule and molecule, and 3) chiral field and molecule. The first method, often called "absolute asymmetric synthesis," employs circularly polarized light as a physical chiral source and a racemic substrate, where no chemical chiral source, such as an optically active reagent or catalyst, is needed. In the second strategy, chiral auxiliary, sensitizer, complexing agent, and solvent have hitherto been employed as chemical chiral sources to induce chirality into a variety of prochiral substrates through intra- or intermolecular chiral interactions in the excited state. The third category, which exploits chiral spatial arrangements and interactions in supramolecular systems, has not extensively been investigated so far, but is a new, intriguing area of photochirogenesis.

From the synthetic point of view, the second strategy using chemical chiral sources is most attractive and promising, and also convenient and practical. Indeed, a great deal of effort has been devoted to the enantio- and diastereodifferentiating photochemical reactions, which afford good to excellent enantomeric excesses (ee's) and diastereomeric excesses (de's), using diverse chiral sensitizers and auxiliaries, respectively [2-4]. A more intriguing phenomenon, as revealed by the mechanistic investigation of asymmetric photosensitizations is the inversion of product chirality; induced by the alteration of environmental factors such as temperature [6-13], pressure [14], solvent [15], and substrate concentration [16]. The chirality switching behavior is not only of mechanistic interest but also of particular practical importance, since such a switching enables us to control the chirality and optical yield of the photoproduct simply by maneuvering these entropy-related external factors, affording both enantiomers without using the antipodal chiral sources.

In this paper, we first present several asymmetric photoreactions that are critically affected by diverse intra- and intermolecular interactions and factors, the elucidation of the origin and mechanism of the chirality switching, and finally discuss the crucial and general roles of entropy in thermal, enzy-

\footnotetext{
*Lecture presented at the XVIII ${ }^{\text {th }}$ IUPAC Symposium on Photochemistry, Dresden, Germany, 22-27 July 2000. Other presentations are published in this issue, pp. 395-548.

†Corresponding author: E-mail: inoue@ @ chem.eng.osaka-u.ac.jp
} 
matic, and photochemical asymmetric reactions. Although most of the results and relevant discussion presented here are based on our recent studies on asymmetric photosensitizations, the fundamental concept of entropically controlling the enantio- and diastereodifferentiating processes should be applicable in general to chemical, photochemical, and biological asymmetric reactions.

\section{TEMPERATURE SWITCHING}

An unusual temperature effect upon product ee was first reported for the enantiodifferentiating $Z-E$ photoisomerization of cyclooctene sensitized by optically active (poly)alkyl benzene(poyl)carboxylates [6] (Scheme 1). Upon photosensitization with (-)-tetramenthyl 1,2,4,5-benzenetetracarboxylate in pentane at $25{ }^{\circ} \mathrm{C},(Z)$-cyclooctene $(\mathbf{1 Z})$ smoothly isomerized to the chiral $(E)$-isomer $(\mathbf{1 E})$ in $9.6 \%$ ee in favor of $(R)$-enantiomer. Unexpectedly, the same photoisomerization conducted at $-90{ }^{\circ} \mathrm{C}$ afforded the antipode $(S)-(+)-1 \mathbf{E}$ in $28.5 \%$ ee, with the product chirality being inverted at the critical point called equipodal temperature $\left(T_{0}\right)$.

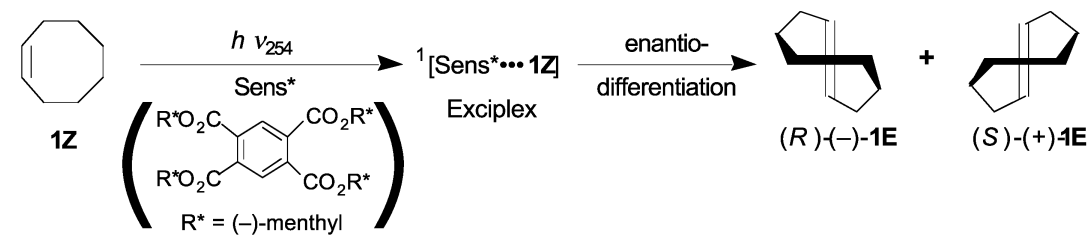

\section{Scheme 1}

It has been demonstrated that this sensitized photoisomerization proceeds through a singlet exciplex ${ }^{1}[$ Sens $* \bullet \bullet 1 Z] ~[17]$ and that the enantiodifferentiation occurs upon rotational relaxation around the double bond of $\mathbf{1 Z}$ within the exciplex intermediate [8]. Thus, the relaxation process should be responsible for the temperature-switching behavior. According to the Eyring treatment, the relative rate constant for the formation of $(S)$ - and $(R)-\mathbf{1 E}, k_{\mathrm{S}} / k_{\mathrm{R}}$, is expressed by eq. 1 .

$$
\ln \left(k_{\mathrm{S}} / k_{\mathrm{R}}\right)=-\Delta \Delta H_{\mathrm{S}-\mathrm{R}}^{\ddagger} / R \cdot T+\Delta \Delta S_{\mathrm{S}-\mathrm{R}}^{\ddagger} / R
$$

where $\Delta \Delta H_{\mathrm{S}-\mathrm{R}}^{\ddagger}$ and $\Delta \Delta S_{\mathrm{S}-\mathrm{R}}^{\ddagger}$ represent the differential activation enthalpy and entropy, respectively. Equation 1 indicates that the logarithm of $k_{\mathrm{S}} / k_{\mathrm{R}}$ is proportional to reciprocal temperature, and that the activation parameters $\left(\Delta \Delta H_{\mathrm{S}-\mathrm{R}}^{\ddagger}\right.$ and $\left.\Delta \Delta S_{\mathrm{S}-\mathrm{R}}^{\ddagger}\right)$ can be calculated from the slope and intercept of the $\ln \left(k_{\mathrm{S}} / k_{\mathrm{R}}\right)$-versus-1/T plot. Hence, the enantiodifferentiating photoisomerization of $\mathbf{1 Z}$ sensitized by a variety of chiral sensitizers was performed at several temperatures to give $\mathbf{1 E}$ in varying ee's, and the $\ln \left(k_{\mathrm{S}} / k_{\mathrm{R}}\right)$ value was plotted against the reciprocal temperature to give an excellent straight line for each sensitizer [6-9]. Typical absolute $\Delta \Delta H_{\text {S-R }}^{\ddagger}$ and $T \Delta \Delta S_{\text {S-R }}^{\ddagger}$ values obtained for non-ortho sensitizers are $<0.1$ and $<0.2 \mathrm{kcal}^{\circ} \mathrm{mol}^{-1}$, respectively, while those for ortho sensitizers are as high as $0.5-1.1$ and $0.5-3.5 \mathrm{kcal}^{\circ} \mathrm{mol}^{-1}$, respectively.

The activation parameters also reveal that the nonzero $\Delta \Delta S_{\text {S-R }}^{\ddagger}$ value, which has the same sign as $\Delta \Delta H_{\mathrm{S}-\mathrm{R}}^{\ddagger}$, is responsible for the temperature switching of product chirality. This is the first asymmetric (photo)reaction in which the vital role of the entropy term has been unambiguously demonstrated experimentally, with accompanying theoretical rationalization. The temperature-switching phenomenon inevitably means that the product ee increases with decreasing temperature only below the equipodal temperature $T_{0}$, while on the contrary the ee increases with increasing temperature above $T_{0}$, which apparently conflicts the conventional belief that higher ee's are obtained in general at lower temperatures. This, however, is a natural consequence of the entropic contribution as can be recognized from the differential Gibbs-Helmholtz eq. 2.

$$
\Delta \Delta G_{\mathrm{S}-\mathrm{R}}^{\ddagger}=\Delta \Delta H_{\mathrm{S}-\mathrm{R}}^{\ddagger}-T \Delta \Delta S_{\mathrm{S}-\mathrm{R}}^{\ddagger}
$$


When the $\Delta \Delta H_{\text {S-R }}^{\ddagger}$ and $T \Delta \Delta S_{\text {S-R }}^{\ddagger}$ values possess the same sign, the differential free-energy change $\left(\Delta \Delta G_{\mathrm{S}-\mathrm{R}}^{\ddagger}\right.$ ) becomes zero at $T=T_{0}$, giving a racemic product; either side of $T_{0}$ their signs are inverted thus switching the product chirality.

Similar temperature-switching behavior has recently been reported for the enantiodifferentiating $Z$-E photoisomerization (and subsequent thermal cyclodimerization) of homologous cycloalkenes such as 1-methylcyclooctene [10], cycloheptene [11], and cyclohexene [12]. However, it should be emphasized that such a dramatic switching of product chirality is not restricted to the unimolecular photoisomerization reactions but is also found in the bimolecular enantiodifferentiating anti-Markovnikov photoaddition of alcohol to 1,1-diphenylpropene sensitized by optically active (di)alkyl naphthalene(di)carboxylates (Scheme 2) [13].



Scheme 2

\section{ROLE OF ORTHO SUBSTITUTION}

It is notable that the temperature switching of product chirality occurs only upon sensitization of $\mathbf{1 Z}$ with chiral ortho-benzenepolycarboxylates, i.e., phthalate, 1,2,4,5-benzenetetracarboxylate, and benzenehexacarboxylate; while the sensitization with meta- and para-benzenepolycarboxylates such as isophthalate, terephthalate, and 1,3,5-benzenetricarboxylate neither exhibit such unusual behavior nor afford an appreciable ee. The unique effect of ortho substitution was further investigated by comparing the enantiodifferentiating photosensitization behavior of hemichiral phthalate and terephthalate, in which one of the sensitizer's chiral moieties, (-)-menthyl in this case, was replaced by a methyl group [8].

Upon photosensitization with hemichiral methyl menthyl terephthalate at several temperatures, the product ee obtained was reduced to exactly half that obtained with dimenthyl terephthalate at each temperature examined. In sharp contrast, hemichiral methyl menthyl phthalate gave an ee almost comparable to, or even higher than, that obtained with dimenthyl phthalate at all temperatures. These contrasting results reveal that the two chiral moieties in terephthalate behave completely independently; whilst the chiral ortho-substituents in phthalate, located closely in space, are no longer independent from each other, and are mutually sterically interacting. This in turn causes dynamic movements synchronized with the rotational relaxation of $\mathbf{1 Z}$ within the exciplex intermediate, resulting in the enormous entropy difference in the transition states $\left(\Delta \Delta S^{\dagger}\right)$.

\section{PRESSURE SWITCHING}

As described above, the product chirality can be controlled, and often inverted, by changing the irradiation temperature. This is made possible through the entropic contribution to the transition state on the excited-state potential surface, which determines the stereochemical consequence of the photoisomerization within the exciplex intermediate. In this context, the pressure effect is an attractive alternative as a controlling factor independent of temperature. Although the pressure effects on various thermochemical and conventional (achiral) photochemical reactions have been studied in considerable detail $[18,19]$, no such effort has been extended to asymmetric photochemistry.

The enantiodifferentiating photoisomerization of $\mathbf{1 Z}$ (Scheme 1) was performed at $25{ }^{\circ} \mathrm{C}$ under high hydrostatic pressures of up to $400 \mathrm{MPa} \bullet$. Analogous to the temperature effect, the product ee was 
highly dependent on the applied pressure only upon sensitization with ortho-benzenepolycarboxylates. For instance, the use of tetramenthyl 1,2,4,5-benzenetetracarboxylate as chiral sensitizer led to a switching of the photoproduct from $(R)-\mathbf{1 E}$ to $(S)-\mathbf{1 E}$ at the equipodal pressure $\left(P_{0}\right)$ of $120 \mathrm{Mpa} \cdot \mathrm{s}$.

The pressure dependence of the product ee, or the relative rate constant $k_{\mathrm{S}} / k_{\mathrm{R}}$ is expressed by eq. 3, as a linear function of pressure $(P)$ at a constant temperature.

$$
\ln \left(k_{\mathrm{S}} / k_{\mathrm{R}}\right)=-\left(\Delta \Delta V_{\mathrm{S}-\mathrm{R}}^{+} / R T\right) P+C
$$

where $\Delta \Delta V^{\ddagger} S-R$ represents the differential activation volume and $C$ the $\ln \left(k_{\mathrm{S}} / k_{\mathrm{R}}\right)$ value at $P=0$. Hence, if the $\ln \left(k_{\mathrm{S}} / k_{\mathrm{R}}\right)$ value and the constant $C$ have the same sign, the product chirality should be inverted at a finite pressure $\left(P_{0}\right)$ as observed above. The $\Delta \Delta V_{\mathrm{S}-\mathrm{R}}^{\star}$ obtained for the ortho-benzenepolycarboxylates are exceptionally large $\left(-5.6\right.$ to $\left.+3.5 \mathrm{~cm}^{3} \cdot \mathrm{mol}^{-1}\right)$ as differential parameters, in view of the non-differential $\Delta V^{\ddagger}$ values reported for the thermal $Z-E$ isomerization of indigo derivatives $\left(-0.6\right.$ to $\left.+9.3 \mathrm{~cm}^{3} \cdot \mathrm{mol}^{-1}\right)$ [20] and for the photochemical [2+2] and [4+2] photocycloaddition reactions $(0$ to +2 and +9 to +12 $\mathrm{cm}^{3} \cdot \mathrm{mol}^{-1}$, respectively) $[19 \mathrm{~b}, 19 \mathrm{c}]$.

From the further pressure-dependence studies performed at lower temperatures, we were able to construct the first three-dimensional $1 / T-P-\ln \left(k_{\mathrm{S}} / k_{\mathrm{R}}\right)$ diagram that correlates the product ee with temperature and pressure [14].

\section{SOLVENT SWITCHING}

More recently, we have found that even the solvent can switch the product chirality in enantiodifferentiating photoisomerization of $\mathbf{1 Z}$ [15]. Although the product ee obtained upon sensitization with chiral polyalkyl benzenepolycarboxylates was not appreciably influenced by the solvent used (pentane, ether, acetonitrile, and methanol) [8,15], the protected saccharide esters of benzenepolycarboxylic acids led to a dramatic switching of the product chirality at low temperatures, just by changing the solvent from pentane to ether [15]. Thus, sensitization with (-)-tetrakis(1,2;5,6-di- $O$-isopropylidene- $\alpha$-D-glucofranyl) [DAG] 1,2,4,5-benzenetetracarboxylate afforded $(R)-\mathbf{1 E}$ in $5.5 \%$ ee both in pentane and in ether at $25{ }^{\circ} \mathrm{C}$. To our surprise, upon lowering the irradiation temperature from $25^{\circ} \mathrm{C}$, the ee value obtained in these two solvents started to deviate in opposite directions, affording the antipodal $(R)-\mathbf{1 E}$ and $(S)-1 \mathbf{E}$ in $40 \%$ and $50 \%$ ee in pentane and ether at $-78{ }^{\circ} \mathrm{C}$, respectively.

Furthermore, comparative photosensitization experiments using menthyl and DAG benzenetetracarboxylates were carried out at $-78{ }^{\circ} \mathrm{C}$ in pentane-ether mixed solvents of various compositions. Although the sensitization with the menthyl ester gave practically the same ee over the whole solvent composition, the ee obtained with the DAG ester was to possess a critical, nonlinear dependency on the ether content; varying rapidly from $40 \%$ ee (in favor of $(R)$-1E) in pure pentane to $0 \%$ ee at an ether content as low as $8 \%$, ultimately reaching a plateau of $50 \%$ ee (in favor of $(S)$-1E) in $50 \%$ ether. Fluorescence intensity from the DAG ester showed a similar nonlinear profile upon changing the mixing ratio of the two solvents [21]. These photochemical and photophysical results jointly indicate that the preferential interaction of polar solvents such as ether with the saccharide moiety of sensitizer in the excited state is responsible for the solvent switching of product chirality. It is interesting to note that even weak solvation of the chiral moiety through the dipole-dipole interaction can influence and switch the sterochemical outcome of the enantiodifferentiating interactions in the excited state.

\section{SWITCHING BY SUBSTRATE CONCENTRATION}

For more intimate and stereochemically well-defined interactions in the excited state, we introduced a chromophoric benzoate group to cyclooctene at the 3-position [16]. This modification affords a chiral substrate, 3-benzoyloxycyclooctene (2Z), photoisomerization of which produces diastereomeric isomers of the $(E)$-form, i.e., $(E)$-2E. Although we originally expected a facile intramolecular photosensitization upon excitation of the benzoate moiety, the excited benzoate did not efficiently transfer its ener- 
gy intramolecularly to the olefinic moiety, but was instead quenched intermolecularly by other $\mathbf{2 Z}$ molecules. Interestingly, the intra- and intermolecular sensitizations gave the opposite diastereomers, i.e., $\left(1 R^{*}, 3 R^{*}\right)$ - and $\left(1 S^{*}, 3 R^{*}\right)-\mathbf{2 E}$ in $17 \%$ and $21 \%$ de, respectively. Since the intra- and intermolecular sensitization processes are competing in rate with each other, we can control the product de and switch the predominant diastereomer by changing the substrate concentration. A plausible rationalization for this unprecedented diastereomer switching by concentration is that the diastereoface of the olefinic part, which is preferred upon intramolecular energy transfer, is conversely hindered by the benzoate substituent upon intermolecular sensitization [16].

Although no enantiomer or diastereomer switching was observed and the obtained ee's were very low, the substrate concentration also determines the product ee upon sensitization of $\mathbf{1 Z}$ with optically active (-)-menthyl benzyl ether and (-)-dimenthyl ortho-xylylene ether [22]. In this case, the excited singlet sensitizer is quenched only at high substrate concentrations $\left(>50 \mathrm{mmol} \cdot \mathrm{dm}^{-3}\right)$ giving $\mathbf{1 E}$ in appreciable $2.5 \%$ ee, while the triplet sensitization dominates at low concentrations affording racemic $\mathbf{1 E}$.

Since multiple, often competing, excited states, intermediates, and/or processes of different lifetimes or rates are simultaneously or successively involved in most photochemical reactions, the present strategy of concentration control should be more widely examined in other enantio- and diastereodifferentiating photochemical reactions.

\section{CONCLUSIONS}

We have demonstrated that the stereochemical outcome of photochirogenic reactions is not only affected by internal factors such as the sensitizer chromophore, chiral substitutent, and substrate structure; but also is critically governed by several external perturbants such as temperature, pressure, solvent, and substrate concentration, which in several cases can even switch the product chirality. The origin of such dynamic behavior of product chirality and ee is exclusively entropic in nature. In the photoisomerization of $\mathbf{1 Z}$ with various chiral sensitizers, the stereochemistry of the photoproduct obtained at temperatures lower than $T_{0}$ is perfectly correlated with that of chiral substituent employed, and is anticipated from steric hindrance considerations [5]. Above $T_{0}$, the entropic contribution surpasses the enthalpic factor, resulting in the inversion of product chirality. It is important, however, to emphasize that the essential enantiodifferentiation mechanism is not altered by any of these external perturbants or by the internal factors such as sensitizer chromophore, chiral auxiliary and substrate, since the compensatory enthalpy-entropy plot, using all the differential activation parameters for the enantiodifferentiating photoisomerizations of $\mathrm{C}_{6}-\mathrm{C}_{8}$ cycloalkenes and 1,3-cyclooctadiene, gives an excellent straight line with an isokinetic temperature of $26^{\circ} \mathrm{C}$ [12].

Although the product-chirality switching and temperature-dependent ee were originally found in the enantiodifferentiating photosensitization, such apparently unusual behavior is not restricted to the excited-state asymmetric reactions but can be found widely in such processes that are governed by weak interactions, irrespective of the electronic state involved. Indeed, similar phenomena have recently been reported in some catalytic [23] and enzymatic [24,25] asymmetric reactions.

In such entropy-controlled systems the product ee, which is often not particularly good under the ambient conditions, is highly sensitive to the external factors. Hence, it is important to fine-tune the reaction conditions by using the multiple entropy-related factors. We may define this type of reactivity/selectivity control via the manipulation of weak interactions as "entropy chemistry."

\section{ACKNOWLEDGMENTS}

The studies have been done at Himeji Institute of Technology, Osaka University, and the Inoue Photochirogenesis Project, ERATO, JST. We gratefully acknowledge the collaborations of all past and present group members quoted in the references. We also thank Dr. Guy A. Hembury for assistance in the preparation of this manuscript.

(C) 2001 IUPAC, Pure and Applied Chemistry 73, 475-480 


\section{REFERENCES}

1. H. Rau. Chem. Rev. 83, 535-547 (1983).

2. Y. Inoue. Chem. Rev. 92, 741-770 (1992).

3. J.-P. Pete. Adv. Photochem. 21, 135-216 (1996).

4. S. R. L. Everitt and Y. Inoue. Organic Molecular Photochemistry, V. Ramamurthy and K. S. Schanze (Eds)., pp. 71-130, Marcel Dekker, New York (1999).

5. Y. Inoue, T. Wada, S. Asaoka, H. Sato, J.-P. Pete. Chem. Commun. 251-259 (2000).

6. Y. Inoue, T. Yokoyama, N. Yamasaki, A. Tai. Nature 341, 225-256 (1989).

7. Y. Inoue, T. Yokoyama, N. Yamasaki, A. Tai. J. Am. Chem. Soc. 111, 6480-6482 (1989).

8. Y. Inoue, N. Yamasaki, T. Yokoyama, A. Tai. J. Org. Chem. 57, 1332-1345 (1992).

9. Y. Inoue, N. Yamasaki, T. Yokoyama, A. Tai. J. Org. Chem. 58, 1011-1018 (1993).

10. H. Tsuneishi, T. Hakushi, A. Tai, Y. Inoue. J. Chem. Soc., Perkin Trans. 2 2057-2062 (1995).

11. R. Hoffmann and Y. Inoue. J. Am. Chem. Soc. 121, 10702-10710 (1999).

12. S. Asaoka, H. Horiguchi, T. Wada, Y. Inoue. J. Chem. Soc., Perkin Trans. 2 737-747 (2000).

13. S. Asaoka, T. Kitazawa, T. Wada, Y. Inoue. J. Am. Chem. Soc. 121, 8486-8498 (1999).

14. Y. Inoue, E. Matsushima, T. Wada. J. Am. Chem. Soc. 120, 10687-10696 (1998).

15. Y. Inoue, H. Ikeda, M. Kaneda, T. Sumimura, S. R. L. Everitt, T. Wada. J. Am. Chem. Soc. 122, 406-407 (2000).

16. T. Inoue, K. Matsuyama, Y. Inoue. J. Am. Chem. Soc. 121, 9877-9878 (1999).

17. Y. Inoue, S. Takamuku, Y. Kunitomi, H. Sakurai. J. Chem. Soc., Perkin Trans. 21672 (1980).

18. Pressure effects on thermal and some photochemical reactions have been reviewed in detail. (a) T. Asano and W. J. le Noble. Chem. Rev. 78, 407 (1978); (b) R. van Eldik, T. Asano, W. J. le Noble. Chem. Rev. 89, 549-688 (1989).

19. Pressure effects on achiral photochemical reactions were also reported. (a) L. A. Brey, G. B. Schuster, H. G. Drickamer. J. Am. Chem. Soc. 101, 129-134 (1979); (b) W. S. Chung, N. J. Turro, J. Mertes, J. Mattay. J. Org. Chem. 54, 4881-4887 (1989); (c) M. Buback, J. Bünger, L. F. Tietze. Chem. Ber. 125, 2577-2582 (1992).

20. Y. Sueishi, K. Ohtani, N. Nishimura. Bull. Chem. Soc. Jpn. 58, 810 (1985).

21. H. Ikeda, T. Wada, Y. Inoue. Manuscript in preparation.

22. H. Tsuneishi, T. Hakushi, Y. Inoue. J. Chem. Soc., Perkin Trans. 2 1601-1605 (1996).

23. J. Otera, K. Sakamoto, T. Tsukamoto, A. Orita. Tetrahedron Lett. 39, 3201-3204 (1998).

24. R. S. Phillips. Trends Biotechnol. 14, 13-16 (1996).

25. H. Hirata, M. Miyagishi, M. Mayama. Nippon Kagaku Kaishi 113-118 (1998). 